\title{
A RANDOM HIERARCHICAL LATTICE: THE SERIES-PARALLEL GRAPH AND ITS PROPERTIES
}

\author{
BEN HAMBLY, ${ }^{*}$ University of Oxford \\ JONATHAN JORDAN, ${ }^{* *}$ University of Sheffield
}

\begin{abstract}
We consider a sequence of random graphs constructed by a hierarchical procedure. The construction replaces existing edges by pairs of edges in series or parallel with probability $p$ and $1-p$ respectively. We investigate the effective resistance across the graphs, first-passage percolation on the graphs and the Cheeger constants of the graphs as the number of edges tends to infinity. In each case we find a phase transition at $p=\frac{1}{2}$.

Keywords: hierarchical lattice, effective resistance, phase transition, first-
\end{abstract} passage percolation, Cheeger constants

AMS 2000 Subject Classification: Primary 60K35

Secondary 05C80;82B20

\section{Introduction and preliminaries}

\subsection{Introduction}

We examine the properties of a specific example from a broad class of graphs constructed by a random hierarchical procedure. Hierarchical lattices (we take the term lattice from the physics literature where it is equivalent to graph) were introduced in the physics literature as simplified structures for investigating various statistical mechanical problems, [4], [5], [6], [7]. They provide approximations to the standard $d$-dimensional lattice but with a much simpler connection structure, which sometimes

* Postal address: Mathematical Institute, University of Oxford, 24-29 St. Giles, Oxford OX1 3LB, UK

** Postal address: Department of Probability and Statistics, University of Sheffield, Hicks Building, Sheffield S3 7RH, UK 
allows exact calculations to be done.

A standard example is the diamond lattice, which is constructed by replacing each edge in the current graph by a diamond, that is four edges, consisting of two pairs of edges in series, connecting the end points of the original edge. Other examples are pre-fractal graphs such as those associated with the Sierpiński gasket and other nested fractals, [2].

The properties of hierarchical lattices can typically be described mathematically by a dynamical system on probability measures. We call this a random hierarchical system. Let $\left(X_{j}^{(0)}\right)_{j \in \mathbb{N}}$ be a sequence of independent identically distributed real valued random variables and define $\left(X_{j}^{(n)}\right)_{j \in \mathbb{N}}$ to be the sequence defined recursively by setting

$$
X_{j}^{(n)}=f\left(X_{k(j-1)+1}^{(n-1)}, X_{k(j-1)+2}^{(n-1)}, \ldots, X_{k j}^{(n-1)}\right)
$$

for some (non-linear) function $f: \mathbb{R}^{k} \rightarrow \mathbb{R}$. This function is usually determined by the structure of the lattice. For example we may be interested in the global resistance across the $n$-th lattice where the $X_{j}^{(0)}$ represent the resistances of each edge and $f$ the effective resistance between the end points. Results about such iterations, for instance the behaviour of $X_{1}^{(n)}$ as $n \rightarrow \infty$, are obtained in [8, 10, 13, 14, 15]. In this setting it has been shown that there is a strong law of large numbers provided the function $f$ satisfies certain conditions.

We wish to extend this to the case where the hierarchical lattice is itself random. The hierarchical systems which describe the properties of such lattices could be called random hierarchical systems in random environment. These lattices provide models for disordered lattices, a more complex setting for doing statistical mechanics. This type of random environment on regular hierarchical lattices is a natural setting for some computer science applications such as the biased coin problem [1]. We will regard the input variables $\left(X_{j}^{(0)}\right)_{j \in \mathbb{N}}$ as either random or deterministic and then, to generate the random environment for our random hierarchical system, we choose the function $f$ to be applied to the random variables randomly. We will obtain distributional fixed points for some such iterations.

As the range of possible lattices and systems generated in this way is vast we restrict ourselves to a particular random hierarchical lattice model which exhibits a range of random hierarchical systems. The specific model will be called the series-parallel graph. 


\subsection{The construction of the series-parallel graph}

We set a parameter $p \in[0,1]$ and define a sequence of graphs $G^{(n)}$. The first graph $G^{(0)}$ consists of two vertices connected by a single edge, with the boundary of $G^{(0)}$ considered to consist of these two vertices.

To construct $G^{(n)}$ from $G^{(n-1)}$, we replace each edge, independently of the structure of $G^{(n-1)}$ and of the other edges, by a new graph element. With probability $p$, this is a two-edge path between the end vertices of the original edge, and otherwise (so with probability $1-p)$ it is a pair of parallel edges between the same two vertices. This means that the graph can have multiple edges.

The two boundary vertices of $G^{(n)}$ are inherited from $G^{(n-1)}$.

A typical graph at stage 5 with $p=\frac{1}{2}$ might look like that in Figure 1.

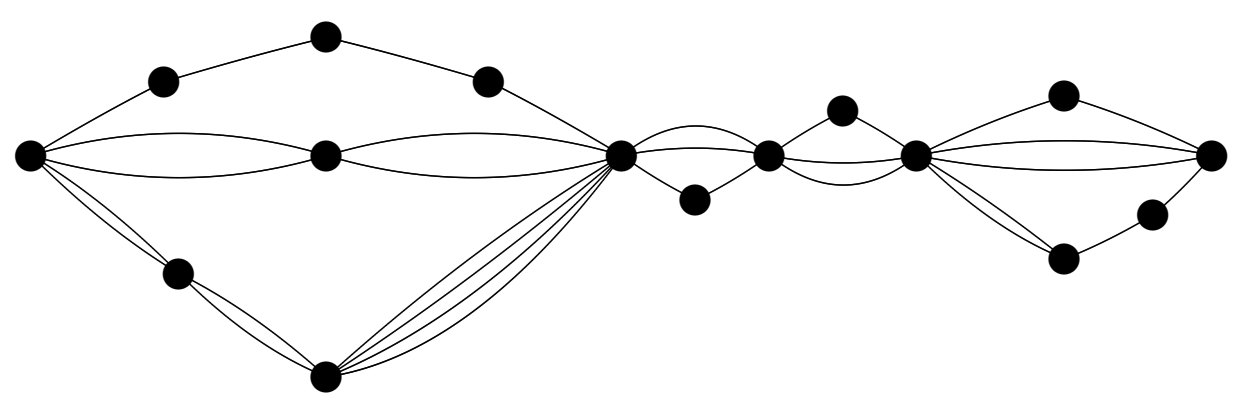

Figure 1: A sample graph, $G^{(5)}$

\subsection{Properties of the series-parallel graph}

The self-similarity of the construction means that, for $n>1$, the graph $G^{(n)}$ is the union of two subgraphs, which we call $G_{1}^{(n-1)}$ and $G_{2}^{(n-1)}$. These correspond to the edges of $G^{(1)}$ and are independent and identically distributed copies of $G^{(n-1)}$, connected together in a manner determined independently by $G^{(1)}$. Hence a property $X^{(n)}$ of $G^{(n)}$ can be thought of as a random function $f$ of the properties $X_{1}^{(n-1)}, X_{2}^{(n-1)}$ of the two graphs $G_{1}^{(n-1)}$ and $G_{2}^{(n-1)}$. Thus the sequence of graphs is a random hierarchical lattice and any property is a random hierarchical system with $k=2, f$ a random function describing the effect on the property $X$ of connecting the two graphs 
together, with initial property $X_{j}^{(0)}$ on edge $j$.

We will be concerned with the limiting properties of this sequence of random graphs. Some properties, such as the resistance and the graph distance between the two boundary points, can also be related to random hierarchical systems with $k=2$ and $f$ a random function related to connecting together the two graphs. There should be evidence of a phase transition in that for $p<\frac{1}{2}$ we see a predominance of parallel edges, while for $p>\frac{1}{2}$ the graphs are closer to a linear graph with some parallel edges.

We discuss a simple property to begin with - the volume of the graph $G^{(n)}$. It is clear by the construction that the number of edges of $G^{(n)}$ is $2^{n}$ and hence we consider the volume to be the number of vertices $V^{(n)}$ in the graph $G^{(n)}$. The random hierarchical system for this example is linear in that

$$
\begin{aligned}
& f_{1}\left(x_{1}, x_{2}\right)=x_{1}+x_{2}-1 \text { with probability } p \\
& f_{2}\left(x_{1}, x_{2}\right)=x_{1}+x_{2}-2 \text { with probability } 1-p .
\end{aligned}
$$

It is easy to see that $2^{-n} V^{(n)} \rightarrow p$ as $n \rightarrow \infty$. Thus there is no evidence of phase transition in this case.

The first non-linear property we will consider is the total effective resistance $R^{(n)}$ between the two boundary points of the graph $G^{(n)}$, considering each edge as a unit resistor. In the random hierarchical system the probability distribution for the random function $f: \mathbb{R}^{2} \rightarrow \mathbb{R}$ is given by

$$
\begin{aligned}
& f_{1}\left(x_{1}, x_{2}\right)=x_{1}+x_{2} \text { with probability } p \\
& f_{2}\left(x_{1}, x_{2}\right)=\left(x_{1}^{-1}+x_{2}^{-1}\right)^{-1} \text { with probability } 1-p .
\end{aligned}
$$

For this example we will see evidence of the phase transition. For $p<\frac{1}{2}$ we show that $R^{(n)} \rightarrow 0$ almost surely, and for $p>\frac{1}{2}$ we show that $R^{(n)} \rightarrow \infty$ almost surely. In the $p=\frac{1}{2}$ case we conjecture that there is no non-degenerate distributional fixed point for the hierarchical system. We also believe that there is an almost sure exponential growth rate for the resistance but can only establish upper and lower bounds on $\log \left(X_{1}^{(n)}\right) / n$ here.

In section 3 , we investigate the limiting behaviour of the graph distance between the two boundary points and first passage percolation on the graphs, which are described by a random hierarchical system which has a non-degenerate fixed point for $p<\frac{1}{2}$. 
Finally, in section 4, we study the behaviour of the Cheeger constants of the graphs, which gives an estimate for the spectral gap and hence the rate of convergence to equilibrium for a random walk on the graph. This also shows the phase transition at $p=\frac{1}{2}$.

\subsection{Branching processes and a zero-one law}

We can define the following branching processes associated with the series-parallel graph.

Definition 1.1. Set $C^{(n)}$ to be the number of individual edges whose removal would disconnect $G^{(n)}$. This behaves as a Galton-Watson branching process with each individual having 2 offspring with probability $p$ and 0 offspring with probability $1-p$.

Similarly set $\tilde{C}^{(n)}$ to be the number $w^{(n)}(1,2)$ of edges connecting the two boundary points of $G^{(n)}$. This also behaves as a branching process, with each individual having 2 offspring with probability $1-p$ and 0 offspring with probability $p$.

The following result will be useful in showing that the probabilities of certain events are either 0 or 1.

Consider an event $A$ determined by the sequence $\left(G^{(n)}\right)_{n \in \mathbb{N}}$ such that if $G^{(1)}$ has a series connection then $A=A_{1} \cup A_{2}$ (where $A_{1}$ and $A_{2}$ are the equivalent events for the

sequences $\left(G_{1}^{(n)}\right)_{n \in \mathbb{N}}$ and $\left.\left(G_{2}^{(n)}\right)_{n \in \mathbb{N}}\right)$, and that if $G^{(1)}$ has a parallel connection then $A=A_{1} \cap A_{2}$.

Lemma 1.1. If $p \neq \frac{1}{2}$, then any such event $A$ must have probability 0 or 1 . This also applies if $\cap$ and $\cup$ are reversed in the above.

Proof. Let $q=\mathbb{P}(A)$. Then $q$ satisfies

$$
q=h(q)=p\left(1-(1-q)^{2}\right)+(1-p) q^{2}=(1-2 p) q^{2}+2 p q
$$

which has roots 0 and 1 unless $p=\frac{1}{2}$ in which case it reduces to $q=q$.

The second statement follows by the same method.

A polynomial $h(q)$ corresponding to $(1-2 p) q^{2}+2 p q$ can be constructed for sequences of graphs derived from similar constructions. In the case where there is just one function (e.g. a deterministic fractal lattice), then the polynomial $h(q)$ is studied in $[11,12]$, 
where it is shown that it has at most one fixed point in $(0,1)$ which will be repulsive. It is also studied in [14]. These results do not apply to randomised constructions, but the fixed points of $h(q)$ still provide useful information.

\section{Limiting behaviour of the resistance}

We begin with an observation about the symmetry of the distribution of the resistance.

Lemma 2.1. The distribution of $R^{(n)}$ with parameter $p$ is the same as the distribution of $1 / R^{(n)}$ with parameter $1-p$.

Proof. Recall that the graph $G^{(n)}$ consists of 2 independent graphs $G_{1}^{(n-1)}, G_{2}^{(n-1)}$, with the same distribution as $G^{(n-1)}$ connected together in a manner determined independently by $G^{(1)}$.

Given $R^{(n)}$ we construct $\tilde{R}^{(n)}$ as follows:

We set $\tilde{R}^{(0)}=R^{(0)}=1$ and define $\tilde{R}_{1}^{(n-1)}$ and $\tilde{R}_{2}^{(n-1)}$ to be independent random variables with the same distribution as $\tilde{R}^{(n-1)}$.

If $R^{(n)}=R_{1}^{(n-1)}+R_{2}^{(n-1)}$, then $\tilde{R}^{(n)}=\frac{\tilde{R}_{1}^{(n-1)} \tilde{R}_{2}^{(n-1)}}{\tilde{R}_{1}^{(n-1)}+\tilde{R}_{2}^{(n-1)}}$.

If $R^{(n)}=\frac{R_{1}^{(n-1)} R_{2}^{(n-1)}}{R_{1}^{(n-1)}+R_{2}^{(n-1)}}$, then $\tilde{R}^{(n)}=\tilde{R}_{1}^{(n-1)}+\tilde{R}_{2}^{(n-1)}$.

By construction $\tilde{R}^{(n)}$ with parameter $p$ is distributed as $R^{(n)}$ with parameter $1-p$.

Now $\tilde{R}^{(0)}={ }_{d} 1 / R^{(0)}$ and assuming (induction hypothesis), that $\tilde{R}^{(n-1)}$ has the same distribution as $1 / R^{(n-1)}$, then if

$$
R^{(n)}=R_{1}^{(n-1)}+R_{2}^{(n-1)},
$$

then

$$
\tilde{R}^{(n)}=\frac{1}{\frac{1}{\tilde{R}_{1}^{(n-1)}}+\frac{1}{\tilde{R}_{2}^{(n-1)}}}={ }_{d} \frac{1}{R_{1}^{(n-1)}+R_{2}^{(n-1)}}=\frac{1}{R^{(n)}}
$$

and also if

$$
R^{(n)}=\frac{R_{1}^{(n-1)} R_{2}^{(n-1)}}{R_{1}^{(n-1)}+R_{2}^{(n-1)}}
$$

then

$$
\tilde{R}^{(n)}=\tilde{R}_{1}^{(n-1)}+\tilde{R}_{2}^{(n-1)}={ }_{d} \frac{1}{R_{1}^{(n-1)}}+\frac{1}{R_{2}^{(n-1)}}=\frac{1}{R^{(n)}} .
$$

Thus $\tilde{R}^{(n)}={ }_{d} 1 / R^{(n)}$ for all $k$. 
Note that the branching process $C^{(n)}$ is a lower bound for the resistance $R^{(n)}$ (as each edge has resistance 1) and its offspring mean is $2 p$, so it is supercritical for $p>\frac{1}{2}$. Thus there is a positive probability of $C^{(n)} \rightarrow \infty$, and hence a positive probability of $R^{(n)} \rightarrow \infty$ (and it grows exponentially as $R^{(n)}>C(2 p)^{n}$ for all $n$, for some $C>0$ ). This can be sharpened to almost surely, but we can prove the following stronger result which will be required in section 4 .

Theorem 2.1. For any sequence of random variables $\left(X^{(n)}\right)_{n \in \mathbb{N}}$, with $X^{(n)}={ }_{d} R^{(n)}$ for each $n$, we have the following.

(1) If $p>\frac{1}{2}$, then $X^{(n)} \rightarrow \infty$ almost surely.

(2) If $p<\frac{1}{2}$, then $X^{(n)} \rightarrow 0$ almost surely.

In order to prove the theorem we require the following technical lemma. We will be interested in the supremum over $x>0$ of

$$
f(x, \beta, p)=\frac{p(1+x)^{\beta}+(1-p)\left(\frac{x}{1+x}\right)^{\beta}}{1+x^{\beta}}
$$

where $\beta=\frac{1}{\alpha}$ for a strictly positive integer $\alpha$.

Lemma 2.2. When $p \leq \frac{1}{2}$ and $\alpha \in \mathbb{N}$, the supremum of $f(x, \beta, p)$ over $x>0$ is attained at $x=1$.

Proof. Differentiate $f(x, \beta, p)$ to get

$$
\begin{gathered}
\frac{\partial f(x, \beta, p)}{\partial x}=\frac{\beta}{\left(1+x^{\beta}\right)^{2}}\left[\left(1+x^{\beta}\right)\left(p(1+x)^{\beta-1}+(1-p)\left(\frac{x}{1+x}\right)^{\beta-1}\left(\frac{1}{1+x}\right)^{2}\right)\right. \\
\left.-x^{\beta-1}\left(p(1+x)^{\beta}+(1-p)\left(\frac{x}{1+x}\right)^{\beta}\right)\right] \\
=\frac{\beta}{\left(1+x^{\beta}\right)^{2}(1+x)^{\beta-1}}\left[p(1+x)^{2 \beta}\left(1-x^{\beta-1}\right)+(1-p) x^{\beta-1}\left(1-x^{2}\right)\right]
\end{gathered}
$$

For $x \leq 1,\left(1-x^{\beta-1}\right) \leq 0$ (because $\left.\beta \leq 1\right)$ and similarly $\left(1-x^{2}\right) \geq 0$. The opposite is the case for $x \geq 1$. If we define

$$
g(x, \beta)=(1+x)^{2 \beta}\left(1-x^{\beta-1}\right)+x^{\beta-1}\left(1-x^{2}\right),
$$

then for $p \leq \frac{1}{2}$ we have for $0<x \leq 1$

$$
\frac{\partial f(x, \beta, p)}{\partial x} \geq \frac{\beta}{2\left(1+x^{\beta}\right)^{2}(1+x)^{\beta+1}} g(x, \beta),
$$


and for $x \geq 1$ we have

$$
\frac{\partial f(x, \beta, p)}{\partial x} \leq \frac{\beta}{2\left(1+x^{\beta}\right)^{2}(1+x)^{\beta+1}} g(x, \beta) .
$$

Hence the sign of $\frac{\partial f(x, \beta, p)}{\partial x}$ is the same as that of $g(x, \beta)$ for all $x>0$. Now, writing

$$
g(x, \beta)=x^{\beta-1}\left[\left(1-x^{2}\right)-(1+x)^{2 \beta}\left(1-x^{1-\beta}\right)\right],
$$

we can also take a factor of $\left(1-x^{\beta}\right)$ out as follows:

$$
\begin{aligned}
g(x, \beta) & =x^{\beta-1}\left(1-x^{\beta}\right)\left[\left(1+x^{\beta}+x^{2 \beta}+\ldots+x^{(2 \alpha-1) \beta}\right)-(1+x)^{2 \beta}\left(1+x^{\beta}+x^{2 \beta}+\ldots+x^{(\alpha-2) \beta}\right)\right] \\
& =x^{\beta-1}\left(1-x^{\beta}\right)\left[\left(1+x^{\beta}+x^{2 \beta}+\ldots+x^{(\alpha-2) \beta}\right)\left(1+x-(1+x)^{2 \beta}\right)+x^{(\alpha-1) \beta}+x^{(2 \alpha-1) \beta}\right] .
\end{aligned}
$$

This shows that when $\alpha \geq 2$ (and so $\beta \leq \frac{1}{2}$ ),

$$
\begin{aligned}
g(x, \beta) & >0 \text { when } 0<x<1 \\
& <0 \text { when } x>1 .
\end{aligned}
$$

Thus $f(x, \beta, p)$ is maximised over $x>0$ by $f(1, \beta, p)$.

For the case $\beta=\alpha=1$ we have $f(x, 1, p)=p+(1-p) \frac{x}{(1+x)^{2}}$, which is also maximised over $x>0$ at $x=1$.

Proof of Theorem 2.1. We will use recurrences for expectation to prove the result. Let $\alpha$ be a strictly positive integer and consider the behaviour of $\left(R^{(n)}\right)^{1 / \alpha}$. Now, by Lemma 2.2,

$$
\begin{aligned}
\mathbb{E}\left(\left(R^{(n)}\right)^{\frac{1}{\alpha}} \mid \mathcal{F}_{n-1}\right) & =p\left(R_{1}^{(n-1)}+R_{2}^{(n-1)}\right)^{\frac{1}{\alpha}}+(1-p)\left(\frac{R_{1}^{(n-1)} R_{2}^{(n-1)}}{R_{1}^{(n-1)}+R_{2}^{(n-1)}}\right)^{\frac{1}{\alpha}} . \\
& =\left(\left(R_{1}^{(n-1)}\right)^{\frac{1}{\alpha}}+\left(R_{2}^{(n-1)}\right)^{\frac{1}{\alpha}}\right) f\left(\frac{R_{2}^{(n-1)}}{R_{1}^{(n-1)}}, \frac{1}{\alpha}, p\right) \\
& \leq f(1,1 / \alpha, p)\left(\left(R_{1}^{(n-1)}\right)^{\frac{1}{\alpha}}+\left(R_{2}^{(n-1)}\right)^{\frac{1}{\alpha}}\right)
\end{aligned}
$$

and hence

$$
\mathbb{E}\left(\left(R^{(n)}\right)^{\frac{1}{\alpha}}\right) \leq 2 f\left(1, \frac{1}{\alpha}, p\right) \mathbb{E}\left(\left(R^{(n-1)}\right)^{\frac{1}{\alpha}}\right)
$$

It is easy to see that if

$$
p<\frac{1}{2^{\frac{1}{\alpha}}+1}
$$

then

$$
2 f(1,1 / \alpha, p)<1 \text {. }
$$


Thus for every $p<1 / 2$ we can choose an $\alpha$ such that

$$
\mathbb{E}\left(\left(R^{(n)}\right)^{1 / \alpha}\right) \leq C^{n}
$$

for a $C=2 f(1,1 / \alpha, p)<1$. Thus, as $X^{(n)}={ }_{d} R^{(n)}$ for all $n,\left(X^{(n)}\right)^{1 / \alpha} \rightarrow 0$ almost surely, using the first Borel-Cantelli Lemma, and hence $X^{(n)} \rightarrow 0$ as required.

The second statement follows immediately from Lemma 2.1 and result (1).

To get some more information about growth or decay rates, we investigate the behaviour of $\mu^{-n} R^{(n)}$. We recall $f(x, \beta, p)$ and define $\epsilon(\alpha)$ such that

$$
\frac{1}{2}+\epsilon(\alpha)=\sup \{p: f(1, \beta, p) \geq f(x, \beta, p) \text { for all } x \geq 0\}
$$

where $\beta=1 / \alpha$. As $f$ is continuous the supremum will be attained.

Lemma 2.3. If $p<\frac{1}{2}+\epsilon(\alpha)$ and

$$
\mu>\left(2 f\left(1, \frac{1}{\alpha}, p\right)\right)^{\alpha}
$$

for some $\alpha \in \mathbb{N}$, then

$$
\mu^{-n} R^{(n)} \rightarrow 0
$$

almost surely and furthermore this applies to any sequence of random variables with these distributions.

Proof. This follows from the fact that

$$
\mathbb{E}\left(\sqrt[\alpha]{\mu^{-n} R^{(n)}}\right) \leq\left(2 \mu^{-\frac{1}{\alpha}} f\left(1, \frac{1}{\alpha}, p\right)\right)^{n}
$$

via a standard argument using the first Borel-Cantelli Lemma.

We note that Lemma 2.2 implies that $\epsilon(\alpha) \geq 0$ for $\alpha \in \mathbb{N}$. This is enough to give the following result on the critical case when $p=\frac{1}{2}$.

Corollary 2.1. When $p=\frac{1}{2}$ and $X^{(n)}={ }_{d} R^{(n)}$ for all $n$,

$$
\frac{1}{n} \log X^{(n)} \rightarrow 0 \text { a.s. as } n \rightarrow \infty \text {. }
$$

Proof. Take $\mu>1$. We require, for some $\alpha \in \mathbb{N}$,

$$
\frac{1}{2}\left(\sqrt[\alpha]{2}+\sqrt[\alpha]{\frac{1}{2}}\right)<\mu^{\frac{1}{\alpha}}
$$


but

$$
\lim _{\alpha \rightarrow \infty}\left(\frac{1}{2}\left(\sqrt[\alpha]{2}+\sqrt[\alpha]{\frac{1}{2}}\right)\right)^{\alpha}=1,
$$

so for sufficiently large $\alpha$ we will have $\mu^{-n} X^{(n)} \rightarrow 0$ almost surely, from Lemma 2.3.

We use the symmetry properties to obtain, for $\mu<1, \mu^{-n} X^{(n)} \rightarrow \infty$ a.s., which gives the result.

Lemma 2.4. We have that

$$
\epsilon(\alpha)>0 \text { for all } \alpha \in \mathbb{N} \text {. }
$$

Proof. We know that $\epsilon(1)=\frac{1}{2}$. Let $\hat{f}(x, \beta, p)=f(x, \beta, p)-f(1, \beta, p)$. From the proof of Lemma 2.2 we see that $\hat{f}(x, \beta, p)$ is strictly negative when $p=\frac{1}{2}, \beta=1 / \alpha, \alpha \in$ $\mathbb{N}$, except when $x=1$. We also note that $\hat{f}(x, \beta, p)$ is infinitely differentiable in $x$ and $p$ (if $x>0)$, and has $\hat{f}(1, \beta, p)=0$ and $\frac{\partial \hat{f}}{\partial x}(1, \beta, p)=0$ for all $\beta, p$.

In order to establish that, for each $\alpha \in \mathbb{N}, \hat{f}(x, 1 / \alpha, p) \leq 0$ for all $x$, for some $p>\frac{1}{2}$, it remains to show that

$$
\frac{\partial^{2} \hat{f}}{\partial^{2} x}\left(1, \beta, \frac{1}{2}\right)<0
$$

so that this second derivative will remain negative for $p$ in a neighbourhood of $\frac{1}{2}$.

A simple calculation gives

$$
\frac{\partial^{2} \hat{f}}{\partial^{2} x}\left(1, \beta, \frac{1}{2}\right)=\frac{\beta}{2^{\beta+4}}\left(2^{2 \beta}(1-\beta)-\beta-1\right) .
$$

We know $\frac{\partial^{2} \hat{f}}{\partial^{2} x}\left(1, \beta, \frac{1}{2}\right) \leq 0$, so we have the result as long as there are no solutions of

$$
\beta=\frac{2^{2 \beta}-1}{2^{2 \beta}+1}
$$

in $(0,1)$. This is also straightforward as

$$
\frac{\mathrm{d}}{\mathrm{d} \beta}\left(\frac{2^{2 \beta}-1}{2^{2 \beta}+1}\right)=\frac{2^{2 \beta+2} \log 2}{\left(2^{2 \beta}+1\right)^{2}}
$$

and hence, as $\frac{4 y}{(y+1)^{2}}<1$ for $y>1$, the derivative is bounded above by $\log 2$ in $(0,1)$, giving the result.

Corollary 2.2. If $X^{(n)}={ }_{d} R^{(n)}$ for all $n$, then

a) For $p>\frac{1}{2}$, there exists a value $\lambda(p)>0$ such that, almost surely,

$$
(2 p-1) \log 2 \leq \frac{1}{n} \log X^{(n)} \leq \lambda(p) \text { eventually. }
$$


Furthermore, $\lambda(p) \rightarrow 0$ as $p \downarrow \frac{1}{2}$.

b) For $p<\frac{1}{2}$,

$$
(2 p-1) \log 2 \geq \frac{1}{n} \log X^{(n)} \geq-\lambda(1-p) \text { eventually. }
$$

Proof. Upper bound of part a):

We use Lemma 2.3 to see that, if $p<\frac{1}{2}+\epsilon(\alpha)$ and

$$
\mu>\left(p \sqrt[\alpha]{2}+(1-p) \sqrt[\alpha]{\frac{1}{2}}\right)^{\alpha}
$$

then

$$
\mu^{-n} X^{(n)} \rightarrow 0 \text { a.s. as } n \rightarrow \infty \text {. }
$$

Thus we can define

$$
\lambda(p)=\inf _{p<\frac{1}{2}+\epsilon(\alpha)} \alpha \log \left(p \sqrt[\alpha]{2}+(1-p) \sqrt[\alpha]{\frac{1}{2}}\right) .
$$

Now we let $p \downarrow \frac{1}{2}$ and use Lemma 2.4 to allow us to take the limit as $\alpha \rightarrow \infty$. The lower bound of part b) follows by symmetry.

Upper bound of part b):

For all $p<\frac{1}{2}$ and $\mu>\left(p \sqrt[\alpha]{2}+(1-p) \sqrt[\alpha]{\frac{1}{2}}\right)^{\alpha}$ we have $\mu^{-n} X^{(n)} \rightarrow 0$ a.s. as $n \rightarrow \infty$. Take the limit of $\mu$ as $\alpha \rightarrow \infty$, using l'Hôpital's rule, to get the result.

The lower bound of part a) follows by symmetry.

We remark that a more detailed calculation, given in [9], shows that when $p=1 / 2$ we have $\mathbb{E}\left(\log X^{(n)}\right)^{2} \rightarrow \infty$ as $n \rightarrow \infty$. We conjecture that the limiting probability measure in the critical case puts mass $1 / 2$ at 0 and $\infty$.

Another natural question that we have not been able to settle is does there exist $\theta(p)$ such that $\lim _{n \rightarrow \infty} \frac{1}{n} \log X^{(n)}=\theta(p)$ ?

\section{The length of the graph}

\subsection{Introduction}

We consider the graph distance between the two boundary points. To study this, we label the vertices as follows: the initial vertices are 1 and 2 , and new vertices are numbered in order of their insertion i.e. when an edge $a \leftrightarrow b$ is replaced by two edges 
in series these are $a \leftrightarrow c$ and $c \leftrightarrow b$ with $c>a, b$. We will define $j(c)$ to be the stage at which vertex $c$ was added to the graph i.e. $j(c)=\min _{n}\left\{c \in V\left(G^{(n)}\right)\right\}$ and further define $n(c)$ to be a neighbour of $c$ in $G^{(j(c))}$ i.e. $n(c)=\min _{a}\left\{a \leftrightarrow c\right.$ in $\left.G^{(j(c))}\right\}$. We take $n(1)=2$ and $n(2)=1$, and $j(1)=j(2)=0$.

We define the distance $d^{(n)}(a, b)$ to be the graph distance in $G^{(n)}$ between $a$ and $b$, for $a, b \in V\left(G^{(n)}\right)$.

We now define $K^{(n)}=d^{(n)}(1,2)$, the distance between the two boundary points. This can be thought of as the 'length' of the graph.

In the hierarchical system framework, $f$ is again chosen randomly from the two functions

$$
\begin{aligned}
& f_{1}\left(x_{1}, x_{2}\right)=x_{1}+x_{2} \text { with probability } p \\
& f_{2}\left(x_{1}, x_{2}\right)=\min \left(x_{1}, x_{2}\right) \text { with probability } 1-p .
\end{aligned}
$$

We define $\kappa_{p}$ to be the map on probability measures $\nu$ on $\mathbb{R}^{+}$associated with this random hierarchical system. That is, given a probability measure $\nu$ on $\mathbb{R}^{+}$, we can take two independent random variables $X_{1}$ and $X_{2}$ with law $\nu$, and an independent uniform $[0,1]$ random variable $Z$, and define a random variable

$$
X= \begin{cases}X_{1}+X_{2} & Z \leq p \\ \min \left(X_{1}, X_{2}\right) & Z>p\end{cases}
$$

setting $\kappa_{p}(\nu)$ to be the law of $X$.

Then, if the random variables $K^{(0)}$ have law $\nu^{(0)}$, the random variables $K^{(n)}$ have law $\kappa_{p}^{n}\left(\nu^{(0)}\right)$.

Lemma 3.1. a) If $p>\frac{1}{2}$, then $K^{(n)}$ grows exponentially quickly with probability 1 .

b) If $p<\frac{1}{2}$, then $K^{(n)}$ has a finite limit $K$ as $n \rightarrow \infty$, with probability 1 .

Proof. Recall the branching processes defined in Definition 1.1.

For a), we use the branching process $C^{(n)}$ from Definition 1.1 to show that the probability is positive, because $K^{(n)} \geq C^{(n)}$.

Now, the method of construction of the graphs ensures that any path of length $k$ between the boundary vertices of $G^{(n)}$ is derived from a path of length $\leq k$ between the boundary vertices of $G^{(n-1)}$, and hence $\left(K^{(n)}\right)_{n \in \mathbb{N}}$ is a non-decreasing sequence. 
Thus the event $\left\{K^{(n)} \rightarrow \infty\right\}$ satisfies the conditions of Lemma 1.1, and therefore it occurs with probability 1.

For b), we use the other branching process $\tilde{C}^{(n)}$ which is the number of edges $1 \leftrightarrow 2$. This is supercritical when $p<\frac{1}{2}$, so with positive probability we have $K^{(n)}=1$ for all $n$. Hence $\mathbb{P}\left(K^{(n)} \rightarrow \infty\right)<1$ and so, applying Lemma 1.1 again on $\left\{K^{(n)} \rightarrow \infty\right\}$, $\mathbb{P}\left(K^{(n)} \rightarrow \infty\right)=0$.

As $\left(K^{(n)}\right)$ is a non-decreasing sequence, the fact that $K^{(n)} \nrightarrow \infty$ almost surely implies that $\lim _{n \rightarrow \infty} K^{(n)}$ has some finite limit $K$ as $n \rightarrow \infty$ almost surely, as required.

Corollary 3.1. When $p<\frac{1}{2}$, the map $\kappa_{p}$ has a non-degenerate fixed point.

Proof. The law of the random variable $K$ gives such a fixed point.

\subsection{The fixed points of $\kappa_{p}$}

We now consider the iteration of $\kappa_{p}$. For $p<\frac{1}{2}$, one fixed point is the distribution $\nu_{p}$ of the random variable $K$ found in Lemma 3.1. We will see that for this range of $p$ this fixed point is essentially unique while for $p>\frac{1}{2}$ there are no non-degenerate fixed points for distributions on $\mathbb{R}^{+}$.

We consider labelling the edges of the graph $G^{(n)}$ with i.i.d. $\mathbb{R}^{+}$-valued random variables with distribution $\mu^{(0)}$. We let $F^{(n)}$ be the minimum sum of the labels on a route between the endpoint vertices. If we regard the random variables as denoting the time taken to cross the edge, the random variable $F^{(n)}$ describes first-passage percolation between the two boundary points of the graph $G^{(n)}$.

Also let $\mu^{(n)}=\kappa_{p}^{n}\left(\mu^{(0)}\right)$.

Lemma 3.2. The distribution of $F^{(n)}$ is $\mu^{(n)}$.

Proof. The statement is obvious for $n=0$. For larger $n$ we assume the statement for $n-1$ and note that $G^{(n)}$ consists of two i.i.d. graphs $G_{1}^{(n-1)}, G_{2}^{(n-1)}$ each with the distribution of $G^{(n-1)}$, that are connected in series with probability $p$ and in parallel with probability $1-p$. In the former case $F^{(n)}$ is the sum of two independent variables with distribution $\mu^{(n-1)}$ and in the latter case it is the minimum of these variables. This describes the map $\kappa_{p}$ so proves the lemma by induction.

We now investigate the sequence $\left(F^{(n)}\right)_{n \in \mathbb{N}}$. We define $\lambda$ to be the infimum of the 
support of $\mu^{(0)}$ i.e. $\lambda=\inf \left\{x: \mu^{(0)}[0, x)>0\right\}$. Note that the law $\mathcal{L}(\lambda K)$ of $\lambda K$ is a fixed point of $\kappa_{p}$ for $p<\frac{1}{2}$.

Theorem 3.1. For $p<\frac{1}{2}$, as $n \rightarrow \infty$,

$$
\kappa_{p}^{n}\left(\mu^{(n)}\right) \rightarrow \mathcal{L}(\lambda K) \text { (weak convergence) }
$$

Proof. We condition on the (replacement) sequence of graphs $G^{(n)}$. As $\left(K^{(n)}\right)_{n \in \mathbb{N}}$ is a non-decreasing sequence in $n$, we know from Lemma 3.1 that for $n$ large enough the 'length' $K^{(n)}=K$. First consider the case where $K=1$. In this case the branching process $\tilde{C}^{(n)}$ from Definition 1.1 grows exponentially almost surely. The value $L^{(n)}$ is bounded above by the minimum label on these $\tilde{C}^{(n)}$ edges. But this minimum value converges weakly to $\lambda$.

Similarly when $K=k$, we consider $n_{0}$ large enough that for all $n \geq n_{0}$ we have $K^{(n)}=d^{(n)}(1,2)=k$. We consider the paths of length $k$ between vertices 1 and 2 in $G^{\left(n_{0}\right)}$, and note that, as there are no paths of length less than $k$ between the boundary vertices of $G^{\left(n_{0}\right)}$ and any path of length $k$ in $G^{(n)}$ for $n>n_{0}$ must be derived from one of length at most $k$ in $G^{\left(n_{0}\right)}, \lim _{n \rightarrow \infty} K^{(n)}=k$ implies that one such path must be preserved in $G^{(n)}$ for $n>n_{0}$. Now, the self-similarity of the structure shows that each edge in this path has a branching process associated with it, with the same offspring distribution as $\tilde{C}^{(n)}$, and also growing exponentially conditioned on survival. Hence the number of edge-disjoint paths from 1 to 2 of length $k$ grows exponentially, and hence the weak convergence result holds.

This shows that $\mathbb{P}\left(F^{(n)}>\lambda K\right) \rightarrow 0$ as $n \rightarrow \infty$. Finally it is obvious that $\mathbb{P}\left(F^{(n)}<\right.$ $\left.\lambda K^{(n)}\right)=0$, completing the proof.

This shows that these are the only fixed points and that starting with any distribution on $\mathbb{R}^{+}$the sequence will converge to one of them.

Note that if $\lambda=0$, the limit is a point mass at 0 .

For $p \geq \frac{1}{2}$ the lower bound in the proof shows that if $\lambda>0$, then $F^{(n)} \rightarrow \infty$ because in that case $K^{(n)} \rightarrow \infty$. Furthermore if $p>\frac{1}{2}$, then $K^{(n)}$ grows exponentially fast almost surely, which is enough to show that the only fixed point is a point mass at 0 .

In the $p<\frac{1}{2}$ case this shows that in the limit first-passage percolation on the graphs $G^{(n)}$ loses all randomness except that coming from the random structure of the graphs. 


\section{Cheeger constants}

In this section we consider the behaviour of the spectral gap for the graphs. We follow [3] to define the eigenvalues of a graph. Given a graph $G$ (which may have multiple edges), we set $V(G)$ to be the set of vertices of $G$. Now for $u, v \in V(G)$ define $\delta(v)$ to be the degree of $v$ in $G$, and $w(u, v)$ to be the number of edges connecting $u$ and $v$. We now define the Laplacian $\Delta$ by

$$
\Delta(u, v)=\left\{\begin{array}{lr}
1 & u=v \\
\frac{-w(u, v)}{\sqrt{\delta(u) \delta(v)}} & u, v \text { adjacent } \\
0 & \text { otherwise }
\end{array}\right.
$$

and we will define the eigenvalues of $G$ to be those of $\Delta$. Let $\lambda_{1}\left(G^{(n)}\right)$ denote the smallest positive eigenvalue of $G^{(n)}$. We anticipate that for $p<\frac{1}{2}$, the graph will have spectral properties similar to those of a compact set, while if $p>\frac{1}{2}$, they will be more like those of the one-dimensional integer lattice.

For a subset $S \subseteq G^{(n)}$, following [3], we define vol $S$ to be the sum of the degrees of vertices in $S$, and define $\bar{S}$ to be the complement of $S$ in $V\left(G^{(n)}\right)$. Further we define $E\left(S_{1}, S_{2}\right)$ to be the number of edges between vertices in $S_{1}$ and vertices in $S_{2}$.

We now define

$$
h_{G^{(n)}}(S)=\frac{|E(S, \bar{S})|}{\min \{\operatorname{vol} S, \operatorname{vol} \bar{S}\}}
$$

and the Cheeger constant

$$
h_{G^{(n)}}=\min _{S} h_{G^{(n)}}(S) .
$$

This is a measure of the connectivity of the graph, see [3] for further details. The Cheeger inequality (proved in [3]) allows us to control the lowest positive eigenvalue (the spectral gap) via the Cheeger constants as

$$
2 h_{G^{(n)}} \geq \lambda_{1}\left(G^{(n)}\right)>\frac{h_{G^{(n)}}^{2}}{2} .
$$

Thus we will consider the asymptotics of the Cheeger constants of the series-parallel graphs $G^{(n)}$.

Lemma 4.1. The Cheeger constant satisfies

$$
\frac{1}{n} \log h_{G^{(n)}} \geq \log \frac{1}{2} .
$$


Furthermore, if $p>\frac{1}{2}$, this bound is asymptotically tight, i.e.

$$
\lim _{n \rightarrow \infty} \frac{1}{n} \log h_{G^{(n)}}=\log \frac{1}{2} .
$$

Proof. As $G^{(n)}$ is connected, $|E(S, \bar{S})| \geq 1$ for any subset $S \subseteq V\left(G^{(n)}\right)$. Furthermore, $\operatorname{vol} G^{(n)}=2^{n+1}$ as there are $2^{n}$ edges in $G^{(n)}$. So $\min \{\operatorname{vol} S, \operatorname{vol} \bar{S}\} \leq 2^{n}$, giving the lower bound.

To prove that it is tight, we aim to prove that for $p>\frac{1}{2}$ there is an almost surely finite random variable $E$ such that, for all $m$, there is a set of edges of size at most $E$ which disconnects the two boundary vertices 1 and 2 . We now proceed in the same way as for Lemma 3.1. We consider the branching process $C^{(n)}$ from Definition 1.1, which is the number of 1-cuts (i.e. edges whose removal would disconnect the graph). This is supercritical when $p>\frac{1}{2}$ so with positive probability $C^{(n)} \rightarrow \infty$, and hence the above statement holds with $E=1$. Letting $E^{(n)}$ be the smallest size of a cut which disconnects the two boundary vertices, we note that $\left(E^{(n)}\right)_{n \in \mathbb{N}}$ is a non-decreasing sequence and that Lemma 1.1 applies to the event $\left\{E^{(n)} \rightarrow \infty\right\}$, so $\left(E^{(n)}\right)_{n \in \mathbb{N}}$ has a finite limit for $p>\frac{1}{2}$ with probability 1 .

Now we let $n_{0}$ be the smallest $n$ such that we can partition the vertex set of $G^{(n)}$ into two sets $S^{(n)}$ and $\bar{S}^{(n)}$ such that $1 \in S^{(n)}$ and $2 \in \bar{S}^{(n)}$ and that each of $S^{(n)}$ and $\bar{S}^{(n)}$ contains an internal edge. The self-similarity of the structure ensures that each edge $e \in E\left(S^{\left(n_{0}\right)}, \bar{S}^{\left(n_{0}\right)}\right)$ has a graph sequence with the same distribution as $G^{(n)}$ associated with it, with an almost surely finite random variable $E_{e}$ corresponding to $E$ above. Hence, for $m>n_{0}$ we can always find a cut of size at most $\sum_{e \in E\left(S^{\left(n_{0}\right)}, \bar{S}^{\left(n_{0}\right)}\right)} E_{e}$ with $S^{(n)} \subseteq S^{(m)}$ and $\bar{S}^{(n)} \subseteq \bar{S}^{(m)}$.

As $S^{\left(n_{0}\right)}$ and $\bar{S}^{\left(n_{0}\right)}$ both contain an interior edge, then vol $S^{(m)}$ and vol $\bar{S}^{(m)}$ will both be at least $\epsilon 2^{m}$ for some $\epsilon>0$ (again using the self-similarity of the structure and the graphs descended from these edges). This is enough to show that $\lim _{n \rightarrow \infty} \frac{1}{n} \log h_{G^{(n)}}=$ $\log \frac{1}{2}$ with probability 1 .

The intuition here is that when $p>\frac{1}{2}$ the structure looks like a relatively small number of long strands, so it is easy to make small cuts. We do not expect this to be true for small $p$ where the graph should be more connected.

The following gives an upper bound on the Cheeger constant when $p \leq \frac{1}{2}$ : 
Lemma 4.2. The sequence of Cheeger constants satisfies

$$
\limsup _{n \rightarrow \infty} \frac{1}{n} \log h_{G^{(n)}} \leq \log \frac{2-p}{2}
$$

almost surely.

Proof. Construct a sequence of sets of vertices $S^{(n)} \subseteq V\left(G^{(n)}\right) ; n \in \mathbb{N}$ as follows: At stage 0 put vertex $1 \in S^{(n)}$, vertex $2 \in \bar{S}^{(n)}$. Then at stage $n+1$ consider each edge of $G^{(n)}$ in $E\left(S^{(n)}, \bar{S}^{(n)}\right)$. The edge will be $a \leftrightarrow b$ with $a \in S^{(n)}$ and $b \in \bar{S}^{(n)}$. If the edge is replaced by a pair of edges in parallel $a \leftrightarrow b$, then keep $a \in S^{(n+1)}$ and $b \in \bar{S}^{(n+1)}$. If the edge is replaced with a pair of edges in series $a \leftrightarrow c$ and $c \leftrightarrow b$, then keep $a \in S^{(n)}$ and $b \in \bar{S}^{(n+1)}$, and place the new vertex $c \in S^{(n)}$ with probability $\frac{1}{2}$.

This ensures that $\frac{1}{n} \log \left(\operatorname{vol} S^{(n)}\right) \rightarrow \log 2$ and $\frac{1}{n} \log \left(\operatorname{vol} \bar{S}^{(n)}\right) \rightarrow \log 2$, almost surely. It is also clear that $\left|E\left(S^{(n)}, \bar{S}^{(n)}\right)\right|$ behaves as a branching process with offspring distribution 1 with probability $p, 2$ with probability $1-p$. Hence $\frac{1}{n} \log \left|E\left(S^{(n)}, \bar{S}^{(n)}\right)\right| \rightarrow$ $\log (2-p)$ almost surely, giving the result.

Lemma 4.3. The sequence of Cheeger constants satisfies

$$
\liminf _{n \rightarrow \infty} \frac{1}{n} \log h_{G^{(n)}} \geq-2 p \log 2 .
$$

Proof. We let $S^{(n)}$ be a subset of the vertex set such that

$$
h_{G^{(n)}}=\frac{\left|E\left(S^{(n)}, \bar{S}^{(n)}\right)\right|}{\operatorname{vol} \bar{S}^{(n)}} .
$$

Note that we may assume that $\bar{S}^{(n)}$ forms a connected subgraph - this is because $\frac{a+b}{c+d} \geq \min \left(\frac{a}{c}, \frac{b}{d}\right)$ for positive $a, b, c, d$ so a disconnected $\bar{S}^{(n)}$ will always contain a connected component giving at least as good a Cheeger constant.

We define $k^{(n)}=\min \left\{a \in \bar{S}^{(n)}\right\}$ and $\hat{k}^{(n)}$ to be its neighbour in $G^{\left(j\left(k^{(n)}\right)\right)}$, i.e. $n\left(k^{(n)}\right)$. We consider the evolution of the edges replacing the edge $\hat{k}^{(n)} \leftrightarrow k^{(n)}$ in $G^{\left(j\left(k^{(n)}\right)\right)}$. (Note that no vertex $c$ with $j(c)<j\left(k^{(n)}\right)$ can be in $\bar{S}^{(n)}$.)

The resistance $R^{(n)}\left(\hat{k}^{(n)}, k^{(n)}\right)$ between $\hat{k}^{(n)}$ and $k^{(n)}$ in $G^{(n)}$ can be bounded below by the reciprocal of the minimum cut between $\hat{k}^{(n)}$ and $k^{(n)}$, which is at least $\frac{1}{\left|E\left(S^{(n)}, \bar{S}^{(n)}\right)\right|}$, and hence

$$
\left|E\left(S^{(n)}, \bar{S}^{(n)}\right)\right| \geq \frac{1}{R^{(n)}\left(\hat{k}^{(n)}, k^{(n)}\right)} .
$$


Due to the connectivity of $\bar{S}^{(n)}$ and the graph structure, the volume satisfies

$$
\operatorname{vol} \bar{S}^{(n)} \leq 2^{n-j\left(k^{(n)}\right)+1}
$$

(any volume larger than this containing $k^{(n)}$ will contain one of the two original (i.e. in $\left.G^{\left(j\left(k^{(n)}\right)\right)}\right)$ neighbours of $k^{(n)}$, which are not in $\bar{S}^{(n)}$ by hypothesis). Thus

$$
h_{G^{(n)}} \geq \frac{1}{2^{n-j\left(k^{(n)}\right)+1} R^{(n)}\left(\hat{k}^{(n)}, k^{(n)}\right)} .
$$

Now

$$
R^{(n)}\left(\hat{k}^{(n)}, k^{(n)}\right)={ }_{d} R^{\left(n-j\left(k^{(n)}\right)\right)}
$$

and we use the complete convergence results of Lemma 2.3 and Corollary 2.2 as follows:

$$
\begin{array}{rll}
h_{G^{(n)}} & \geq_{d} & \frac{1}{2^{n-j\left(k^{(n)}\right)+1} R^{\left(n-j\left(k^{(n)}\right)\right)}} \\
2(2 \mu)^{n-j\left(k^{(n)}\right)} h_{G^{(n)}} & \geq_{d} & \frac{1}{\mu^{-\left(n-j\left(k^{(n)}\right)+1\right)} R^{\left(n-j\left(k^{(n)}\right)\right)}} .
\end{array}
$$

Using Corollary 2.2 we choose $\mu>2^{2 p-1}$ so that

$$
\frac{1}{\mu^{-\left(n-j\left(k^{(n)}\right)+1\right)} R^{\left(n-j\left(k^{(n)}\right)\right)}} \rightarrow \infty \text { a.s. }
$$

as long as $n-j\left(k^{(n)}\right) \rightarrow \infty$ a.s. This condition is satisfied because of the bound above on the volume and the lim sup result for the Cheeger constant in Lemma 4.2.

So, for $\mu>2^{2 p-1}$,

$$
(2 \mu)^{n} h_{G^{(n)}} \geq(2 \mu)^{n-j\left(k^{(n)}\right)} h_{G^{(n)}} \rightarrow \infty
$$

almost surely, because of the complete convergence in Lemma 2.3.

Thus $\log 2 \mu+\liminf _{n \rightarrow \infty} \frac{1}{n} \log h_{G^{(n)}} \geq 0$, which gives the result.

We now put Lemmas 4.1, 4.2 and 4.3 together to obtain the following:

Theorem 4.1. When $p>\frac{1}{2}$,

$$
\lim _{n \rightarrow \infty} \frac{1}{n} \log h_{G^{(n)}}=-\log 2
$$

almost surely.

When $p \leq \frac{1}{2}$,

$$
\liminf _{n \rightarrow \infty} \frac{1}{n} \log h_{G^{(n)}} \geq-2 p \log 2
$$


and

$$
\limsup _{n \rightarrow \infty} \frac{1}{n} \log h_{G^{(n)}} \leq \log \frac{2-p}{2}
$$

also almost surely.

This gives a clear transition in behaviour as $p$ passes through $\frac{1}{2}$.

Finally we can conclude with a statement about the spectral gap.

Corollary 4.1. When $p>\frac{1}{2}$,

$$
-2 \log 2 \leq \liminf _{n \rightarrow \infty} \frac{1}{n} \log \lambda_{1}\left(G^{(n)}\right) \leq \limsup _{n \rightarrow \infty} \frac{1}{n} \log \lambda_{1}\left(G^{(n)}\right) \leq-\log 2
$$

almost surely.

When $p \leq \frac{1}{2}$,

$$
-4 p \log 2 \leq \liminf _{n \rightarrow \infty} \frac{1}{n} \log \lambda_{1}\left(G^{(n)}\right) \leq \limsup _{n \rightarrow \infty} \frac{1}{n} \log \lambda_{1}\left(G^{(n)}\right) \leq \log \frac{2-p}{2},
$$

almost surely.

Acknowledgment The authors would like to thank Neil O'Connell for helpful discussions.

\section{References}

[1] Alon, N. And NaOr, M.(1993) Coin-flipping games immune against linear sized coalitions. SIAM J. Comput., 22, 403-417.

[2] Barlow, M. T. (1998) Diffusions on fractals. École d'été de Saint-Flour XXV (Lecture Notes in Mathematics, 1690). Springer-Verlag, New York.

[3] Chung, F. R. K. (1997). Spectral Graph Theory. No. 92 in CBMS Regional Conference Series. AMS, Providence, Rhode Island.

[4] Cook, J. And Derrida, B. (1989) Polymers on disordered hierarchical lattices: a nonlinear combination of random variables. J. Statist. Phys. 57, 89-139.

[5] Derrida, B. (1986). Pure and random models of statistical mechanics on hierarchical lattices. In Critical Phenomena, Random Systems and Gauge Theories. ed. K. Osterwalder and R. Stora. North-Holland, Amsterdam pp. 989 999. 
[6] Essoh, C. D. And Bellisard, J. (1989). Resistance and fluctuation of a fractal network of random resistors: a non-linear law of large numbers. J. Phys. A 22, $4537-4548$.

[7] Griffiths, R. and Kaufman, M. (1982). Spin systems on hierarchical lattices. Phys. Rev. B 26, 5022-5032.

[8] JoRdAn, J. H. (2002). Almost sure convergence for iterated functions of independent random variables. Ann. App. Prob. 12, 985-1000.

[9] Jordan, J. H. (2003) Renormalisation of random hierarchical systems. D.Phil. thesis, University of Oxford.

[10] Li, D. L. And Rogers, T. D. (1999). Asymptotic behavior for iterated functions of random variables. Ann. App. Prob. 9, 1175-1201.

[11] Moore, E. And Shannon, C. E. (1956). Reliable circuits using less reliable relays, i. J. Franklin Inst. 262, 191-208.

[12] Moore, E. And Shannon, C. E. (1956). Reliable circuits using less reliable relays, ii. J. Franklin Inst. 262, 281-297.

[13] Schenkel, A., Wehr, J. And Wittwer, P. (2000). Computer-assisted proofs for fixed point problems in Sobolev spaces. Elec. J. Math. Phys. 6,.

[14] Shneiberg, I. Y. (1986). Hierarchical sequences of random variables. Theory Probab. Appl. 31, 137-141.

[15] Wehr, J. and Woo, J.-M. (2001). Central limit theorems for nonlinear hierarchical sequences of random variables. J. Stat. Phys. 104, 777-797. 